\title{
Do all patients in the phase I oncology trials need to be hospitalized? Domestic but outstanding issues for globalization of drug development in Japan
}

\author{
Akihiko Shimomura ${ }^{1,2}$ - Shunsuke Kondo ${ }^{1}$ Noriko Kobayashi $^{3} \cdot$ Satoru Iwasa $^{1}$ • \\ Shigehisa Kitano $^{1} \cdot$ Kenji Tamura $^{1,2} \cdot$ Yutaka Fujiwara $^{1} \cdot$ Noboru Yamamoto $^{1,3}$
}

Received: 3 January 2017 / Accepted: 25 February 2017 / Published online: 14 March 2017

(C) The Author(s) 2017. This article is published with open access at Springerlink.com

\begin{abstract}
Introduction Most trials investigating new drugs around the world, including phase I trials, are conducted in outpatient clinics. However, in Japan, regulatory authority requirements and traditional domestic guidelines often require hospitalization of phase I study participants.

Patients and methods Patients participating in single-agent phase I clinical trials at National Cancer Center Hospital between December 1996 and August 2014 were monitored. Toxicity requiring hospitalization is defined as toxicity that needs intensive treatment. Study designs were classified into three types: first-in-human (FIH) study, dose-escalation study (conventional dose-escalation study to determine maximum tolerated dose (MTD) in Japanese patients), and dose-finding study (to assess safety and pharmacokinetic profiles up to the MTD previously determined in the West). Results A total of 945 patients who participated in a variety of single-agent phase I clinical trials between December 1996 and August 2014 were included in this study. Patients participated in one of three study types: dose-escalation $(n=582,62 \%)$, first-in-human $(n=129,14 \%)$, or dosefinding $(n=234,25 \%)$. A total of 76 study drugs were evaluated as part of this pool of phase I studies. Subdivided by mechanism of action, 20 (26\%) were cytotoxic, 50
\end{abstract}

Noboru Yamamoto

nbryamam@ncc.go.jp

1 Department of Experimental Therapeutics, National Cancer Center Hospital, 5-1-1, Tsukiji, Chuo-ku, Tokyo 104-0045, Japan

2 Department of Breast and Medical Oncology, National Cancer Center Hospital, Tokyo, Japan

3 Clinical Trial Support Office, National Cancer Center Hospital, Tokyo, Japan
(66\%) were molecularly targeted, and $6(8 \%)$ were immune checkpoint inhibitor. Thirty-six patients (3.8\%) had severe toxicities requiring hospitalization during the first cycle. The overall number of toxicities requiring hospitalization and/or grade 4 toxicities during any cycle was $5.0 \%$.

Conclusions The frequency of severe toxicity that needs to be hospitalized was unexpectedly low. The data did not demonstrate the need for hospitalization in the phase I trials, suggesting that phase I trials in Japan could be conducted in outpatient settings.

Keywords Phase I trial · Domestic guideline - Severe toxicity $\cdot$ Toxicity management

\section{Introduction}

Phase I clinical trials of oncologic drugs are conducted to determine the proper dosage of both single-agent and combination therapies. If the therapeutic index is very narrow, it is critical to evaluate the study drug toxicity in dose-escalation studies. Dose-limiting toxicity (DLT) is evaluated during the first cycle of each new dosage level for every trial participant to determine the maximum tolerated dose (MTD). Close monitoring for symptoms as well as physiological and laboratory data are required to determine if DLT is observed.

Clinical trials in Japan and Western countries are carried out under the International Conference on Harmonization of Technical Requirements for Registration of Pharmaceuticals for Human Use (ICH) guidelines [1]. These guidelines do not require hospitalization as part of phase I clinical trials. However, according to Japanese guidelines, participants in phase I oncology clinical trials are required to be hospitalized during the first cycle of treatment to observe toxicity closely for safety reasons [2]. 
Because the assessment period for DLT is approximately 1 month in most phase I oncology clinical trials, in Japan study participants are required to be hospitalized for 1 month. Phase I clinical oncology trial participants are generally refractory to standard treatments and usually have very limited options. Even though these patients have a good performance status and generally good health, their life expectancy is limited, and consequently, the phase I hospitalization requirement is sometimes in conflict with ongoing end-oflife care. Additionally, the patient is usually charged with the cost of hospitalization, making a patient's participation in the phase I trial burdensome with respect to time and cost. For all these reasons, it is very difficult to recruit patients to participate in phase I clinical trials. Furthermore, Japan's hospitalization practice makes it an outlier from standard ICH guidelines, limiting the globalization of Japanese oncology drug development.

Toxicity assessment is an essential objective of a phase I trial. Hyman et al. have developed a nomogram-based model to predict the risk of a patient developing a cycle-one serious drug-related toxicity (SDRT), but its practical use remains very limited [3]. Additionally, the type of investigational drugs upon which this model was largely derived has changed from cytotoxic drugs to molecularly targeted drugs and immune checkpoint inhibitors during the past several decades. Consequently, as the mechanism of action of new cancer drugs has changed, the toxicity profiles and timing of toxicity events have also changed. For example, Postel-Vinay et al. reported that in phase I oncology trials of molecularly targeted drugs, treatment interruption or discontinuation occurs more frequently in the second cycle rather than the first cycle [4].

If the frequency of severe toxicity during first cycle in phase I trials is low, it may be more practical to conduct trials in an outpatient setting or with only minimal hospitalization. Furthermore, minimizing or avoiding hospitalization altogether may facilitate recruitment of participants and accelerate clinical drug development. However, to date, there has been no evaluation of the incidence and risk of severe toxicity in hospitalized patients participating in phase I clinical oncology trials in Japan.

In this study, we surveyed severe toxicity observed in the phase I single-agent clinical trials conducted in our institution and investigated the frequency of toxicity that did or did not require hospitalization.

\section{Patients and methods}

\section{Patients}

Patients who participated in single-agent phase I clinical trials at National Cancer Center Hospital between
December 1996 and August 2014 were included in this study. Drug type, treatment course, and toxicity were retrieved from medical records and databases. Severity of toxicity was assessed by Common Terminology Criteria for Adverse Events v. 4.0 [5]. Severe toxicity was classified as requiring hospitalization. Such toxicity requiring hospitalization is defined as follows: toxicity that requires intensive treatment (e.g., continuous oxygen administration, invasive procedure) and/or toxicity that requires intravenous intervention (e.g., fluid therapy, intravenous antibiotics, blood transfusion). Study designs were classified into three types: (1) dose-escalation (conventional doseescalation study to determine MTD in Japanese patients; FIH study was excluded); (2) first-in-human (FIH); and (3) dose-finding (to assess drug safety and pharmacokinetic profiles up to the MTD previously determined in Western studies).

\section{Ethical considerations}

The present study involving human subjects was approved by the National Cancer Center Institutional Review Board (2014-148). A copy of the letter from the Institutional Review Board is available for review by the Editor of this journal.

\section{Results}

A total of 945 patients participated in the phase I trial from December 1996 to August 2014 at the National Cancer Center Hospital (Tokyo, Japan). Median patient age was 58 years (range, 18-76 years); 537 patients (57\%) were men and $408(43 \%)$ were women. Patients were assigned to receive cytotoxic drugs $(n=207,22 \%)$, molecularly targeted drugs $(n=690,73 \%)$, or immune checkpoint inhibitors $(n=48,5 \%)$. Patients participated in one of three study types: dose-escalation $(n=582,61 \%)$, first-inhuman $(n=129,14 \%)$, or dose-finding $(n=234,25 \%)$. Tumor types were non-small cell lung cancer $(n=248$, $28 \%)$, colorectal cancer $(n=175,19 \%)$, sarcoma $(n=115$, $12 \%)$, esophageal cancer $(n=49,5 \%)$, pancreatic cancer ( $n=45,5 \%)$, bile duct cancer $(n=36,4 \%)$, breast cancer $(n=35,4 \%)$, and gastric cancer $(n=26,3 \%)$ (Table 1$)$.

A total of 76 study drugs were evaluated as part of this pool of phase I studies. Subdivided by mechanism of action, $20(26 \%)$ were cytotoxic, $50(66 \%)$ were molecularly targeted, and $6(8 \%)$ were immune checkpoint inhibitor (Table 2).

Ninety-eight patients $(10.4 \%)$ developed severe toxicities during the first cycle; a total of 126 patients $(13.3 \%)$ developed severe toxicities during any cycle. Thirty-six patients $(3.8 \%)$ had severe toxicities requiring 
Table 1 Patient characteristics

\begin{tabular}{ll}
\hline Patients, $n$ & 945 \\
Age, years & 58 (median), 18-76 (range) \\
Sex, $n(\%)$ & \\
Male & $537(57)$ \\
Female & $408(43)$ \\
Drug type, $n(\%)$ & \\
Cytotoxic & $207(22)$ \\
Molecularly targeted & $690(73)$ \\
Checkpoint inhibitor & $48(5)$ \\
Study type, $n(\%)$ & \\
Dose escalation & $582(61)$ \\
First in human (FIH) & $129(14)$ \\
Dose finding & $234(25)$ \\
Disease, $n(\%)$ & \\
NSCLC & $248(26)$ \\
Colorectal & $175(19)$ \\
Sarcoma & $115(12)$ \\
Esophagus & $49(5)$ \\
Pancreas & $45(5)$ \\
Biliary & $36(4)$ \\
Breast & $35(4)$ \\
Gastric & $26(3)$ \\
Melanoma & $23(2)$ \\
Ovarian & $23(2)$ \\
SCLC & $20(2)$ \\
HN & $18(2)$ \\
Prostate & $18(2)$ \\
Uterine & $17(2)$ \\
CUP & $10(1)$ \\
Other & $87(9)$ \\
\hline
\end{tabular}

$N S C L C$ non-small cell lung cancer, $S C L C$ small cell lung cancer, $H N$ head and neck, $C U P$ carcinoma of unknown primary

Table 2 Characteristics of study drugs and clinical studies

\begin{tabular}{ll}
\hline Study drug, $n$ & 76 \\
Study drug, $n(\%)$ & \\
Cytotoxic & $20(26)$ \\
Molecularly targeted & $50(66)$ \\
Checkpoint inhibitor & $6(8)$ \\
Study type, $n(\%)$ & \\
Dose escalation & $44(57)$ \\
First in human & $8(11)$ \\
Dose findings & $24(32)$ \\
\hline
\end{tabular}

hospitalization during the first cycle (Fig. 1). The overall number of toxicities requiring hospitalization and/or grade 4 toxicities during any cycle was $5.0 \%$. In the first cycle, 31 patients $(15.0 \%)$ who received cytotoxic drugs

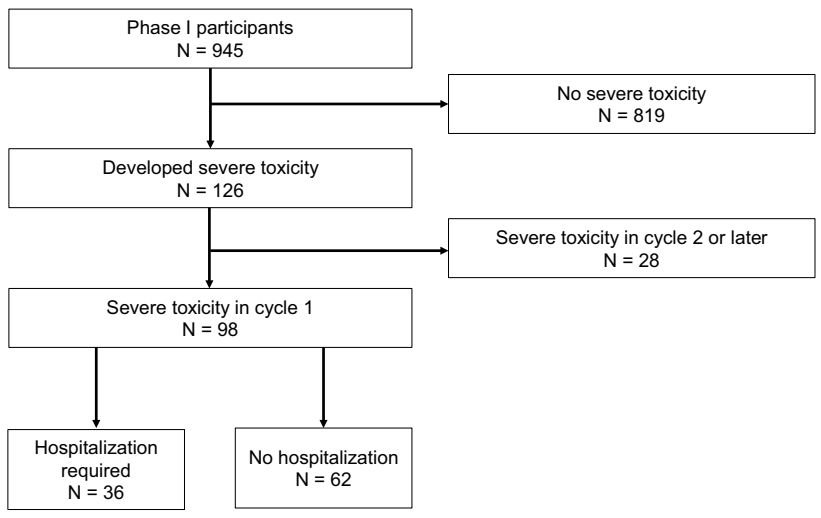

Fig. 1 Patient outcomes

and 67 patients $(9.7 \%)$ who received molecularly targeted drugs developed severe toxicity. However, patients who received immune checkpoint inhibitors did not develop any severe toxicity. Fourteen patients $(6.8 \%)$ receiving cytotoxic drugs and 22 patients $(3.2 \%)$ receiving molecularly targeted drugs needed to be hospitalized because of toxicity. For patients receiving cytotoxic agents, 4 hematological $(1.9 \%)$ and 10 nonhematological $(4.8 \%)$ toxicity events required hospitalization (Table 3 ). For patients receiving molecularly targeted drugs, 3 hematological $(0.4 \%)$ and 19 nonhematological $(2.8 \%)$ toxicity events requiring hospitalization (Table 3 ).

Seventy-three patients $(12.5 \%)$ in dose-escalation studies, 14 patients $(10.9 \%)$ in FIH studies, and 11 patients $(4.7 \%)$ in dose-finding studies developed severe toxicity, including DLT. Twenty-seven (4.6\%) dose-escalation study participants, 4 (3.1\%) FIH study participants, and $5(2.1 \%)$ dose-finding study participants needed hospitalization for toxicity (Table 4).

The observed toxicity profile included increase in aminotransferase $(n=39,17.3 \%)$, neutropenia $(n=22$, $9.7 \%)$, thrombocytopenia $(n=20,8.8 \%)$, anorexia $(n=13,5.8 \%)$, and proteinuria $(n=13,5.8 \%)$. During the first cycle, toxicities requiring hospitalization included thrombocytopenia $(n=11,19.0 \%)$, febrile neutropenia $(n=9,15.5 \%)$, ileus/bowel obstruction $(n=5$, $8.6 \%)$, arrhythmia $(n=3,5.2 \%)$, and pneumonia $(n=3$, $5.2 \%$ ) (Table 5).

\section{Discussion}

Toxicity evaluation is one of the primary objectives of phase I oncology trials because such studies help define the recommended dose for further studies. The type and timing of toxicity can differ depending on a variety of factors including different dose levels, the class and 
Table 3 Toxicity by drug type

\begin{tabular}{|c|c|c|c|c|c|}
\hline & \multicolumn{5}{|c|}{ Drug type } \\
\hline & \multicolumn{2}{|c|}{ Cytotoxic, $n=207$} & \multicolumn{2}{|c|}{$\begin{array}{l}\text { Molecularly targeted, } \\
n=690\end{array}$} & \multirow[t]{2}{*}{ Checkpoint inhibitor, $n=48$} \\
\hline Number of severe toxicities in cycle $1, n(\%)$ & $31(15)$ & & $67(9.7)$ & & \\
\hline \multicolumn{6}{|l|}{ Hospitalization required, $n(\%)$} \\
\hline Hematological & $14(6.8)$ & $4(1.9)$ & $22(3.2)$ & $3(0.4)$ & $0(0)$ \\
\hline Nonhematological & & $10(4.8)$ & & $19(2.8)$ & \\
\hline \multicolumn{6}{|l|}{ No hospitalization required, $n(\%)$} \\
\hline Hematological & $17(8.2)$ & $8(3.9)$ & $45(6.5)$ & $6(0.9)$ & $0(0)$ \\
\hline Nonhematological & & $9(4.3)$ & & $39(5.7)$ & \\
\hline
\end{tabular}

Table 4 Toxicity by study type

\begin{tabular}{llrr}
\hline & Dose escalation, $n=582$ & FIH, $n=129$ & Dose finding, $n=234$ \\
\hline Number of severe toxicities in cycle 1, $n(\%)$ & $73(12.5)$ & $14(10.9)$ & $11(4.7)$ \\
Required hospitalization, $n(\%)$ & $27(4.6)$ & $4(3.1)$ & $5(2.1)$ \\
Required hospitalization and/or G4, $n(\%)$ & $34(5.8)$ & $7(5.4)$ & $6(2.6)$ \\
\hline
\end{tabular}

FIH first-in-human

mechanism of action of the study drug, and the route of administration [6,7]. As new cancer targets have emerged over the past several decades, there is now a much wider array of investigational drugs with new mechanisms of action. As such, classical a " $3+3$ design" may be not sufficient to evaluate toxicities in the current era [7-9].

A modeling approach using toxicity data from 3104 participants in 127 phase I trials between 2000 and 2010 to estimate the risk of SDRT has been reported [3]. In this study, SDRT was defined as grade $\geq 4$ hematological toxicity or grade $\geq 3$ nonhematological toxicity attributed, at least possibly, to study drug(s). The parameters used in this empirical model to predict SDRT risk included performance status, white blood cell count, creatinine clearance, serum albumin, alanine aminotransferase, aspartate aminotransferase, alkaline phosphatase, number of study drug(s), class of study drug, dose level, and constitutional symptoms. Although the resulting nomogram can be useful in predicting a patient's risk for SDRT at the time of enrollment, its usage remains limited. In our study, we assessed every toxicity observed after study enrollment regardless of study drug causality. Frequent severe toxicities were increased aminotransferase, neutropenia, and thrombocytopenia. Toxicities requiring hospitalization were hematological, such as thrombocytopenia and febrile neutropenia, a tendency similar to that previously observed [3]. Toxicity requiring hospitalization was more frequent in studies involving cytotoxic drugs compared to those using molecularly targeted drugs $(15.9 \%$ vs. 9.4\%, respectively) (Table 3). This result is consistent with other reported observations that molecularly targeted drugs are negatively correlated with SDRT. In this study, we did not observe severe toxicity in patients who received immune checkpoint drugs. However, there are some reports of severe toxicity associated with this class of drugs [10], and the lack of observed severe toxicity in our study may be because only a small number of patients received immune checkpoint drugs compared to the other drug classes.

Severe toxicity was observed in $10.4 \%$ of all participants during the first cycle and in $3.2 \%$ of all participants during the second or later cycles, a result similar to a previous report. Thus, it is important to observe toxicity carefully not only during the first cycle but in later cycles as well. We observed that $3.8 \%$ of participants experienced severe toxicity requiring hospitalization, and that $5.0 \%$ of all participants experienced either grade 4 toxicity or severe toxicity requiring hospitalization. This frequency was unexpectedly low and was independent of drug class. This overall low severe toxicity rate suggests that toxicity can be safely monitored in an outpatient setting and that hospitalization during the entire clinical study period is not necessary in most studies. Nonetheless, toxicity observation is still important after the DLT evaluation period. Moreover, it is important to continue careful monitoring for toxicity throughout the course of a phase I trial of immune checkpoint inhibitors because such drugs may have delayed adverse effects [10]. 
Table 5 Adverse events (AEs) observed during trial

\begin{tabular}{|c|c|c|c|c|}
\hline & \multicolumn{2}{|c|}{ Any AEs } & \multicolumn{2}{|c|}{$\begin{array}{l}\text { AEs requiring } \\
\text { hospitalization } \\
\text { in cycle } 1\end{array}$} \\
\hline & $n$ & $\%$ & $n$ & $\%$ \\
\hline Aminotransferase increased & 39 & 17.3 & 4 & 6.9 \\
\hline Neutropenia & 22 & 9.7 & 0 & 0.0 \\
\hline Thrombocytopenia & 20 & 8.8 & 11 & 19.0 \\
\hline Anorexia & 13 & 5.8 & 1 & 1.7 \\
\hline Proteinuria & 13 & 5.8 & 0 & 0.0 \\
\hline Nausea & 10 & 4.4 & 0 & 0.0 \\
\hline Febrile neutropenia & 9 & 4.0 & 9 & 15.5 \\
\hline Anemia & 7 & 3.1 & 2 & 3.4 \\
\hline Malaise & 7 & 3.1 & 1 & 1.7 \\
\hline CPK increased & 5 & 2.2 & 0 & 0.0 \\
\hline Diarrhea & 5 & 2.2 & 0 & 0.0 \\
\hline Ileus & 5 & 2.2 & 5 & 8.6 \\
\hline Rash & 5 & 2.2 & 1 & 1.7 \\
\hline T-Bil increased & 5 & 2.2 & 0 & 0.0 \\
\hline WBC decreased & 5 & 2.2 & 0 & 0.0 \\
\hline Hypertension & 4 & 1.8 & 0 & 0.0 \\
\hline GGT increased & 4 & 1.8 & 0 & 0.0 \\
\hline Mucositis & 4 & 1.8 & 1 & 1.7 \\
\hline Vomiting & 4 & 1.8 & 2 & 3.4 \\
\hline Arrhythmia & 3 & 1.4 & 3 & 5.2 \\
\hline Pneumonitis & 3 & 1.4 & 3 & 5.2 \\
\hline Colitis & 2 & 0.9 & 2 & 3.4 \\
\hline Hypoxia & 2 & 0.9 & 2 & 3.4 \\
\hline Infection & 2 & 0.9 & 2 & 3.4 \\
\hline CIPN & 2 & 0.9 & 0 & 0.0 \\
\hline QTc prolonged & 2 & 0.9 & 0 & 0.0 \\
\hline Unacceptable by patients & 2 & 0.9 & 0 & 0.0 \\
\hline AMY increased & 1 & 0.4 & 0 & 0.0 \\
\hline Constipation & 1 & 0.4 & 1 & 1.7 \\
\hline Creatinine increased & 1 & 0.4 & 0 & 0.0 \\
\hline Dehydration & 1 & 0.4 & 1 & 1.7 \\
\hline Drug fever & 1 & 0.4 & 0 & 0.0 \\
\hline Dry skin & 1 & 0.4 & 0 & 0.0 \\
\hline DVT & 1 & 0.4 & 0 & 0.0 \\
\hline Dyspnea & 1 & 0.4 & 1 & 1.7 \\
\hline ECG abnormality & 1 & 0.4 & 0 & 0.0 \\
\hline Edema & 1 & 0.4 & 0 & 0.0 \\
\hline Hearing disorder & 1 & 0.4 & 0 & 0.0 \\
\hline Hyperglycemia & 1 & 0.4 & 1 & 1.7 \\
\hline Hypoalbuminemia & 1 & 0.4 & 0 & 0.0 \\
\hline Hypotension & 1 & 0.4 & 1 & 1.7 \\
\hline Lipase increased & 1 & 0.4 & 0 & 0.0 \\
\hline Muscle weakness, extremity lower & 1 & 0.4 & 0 & 0.0 \\
\hline Perforation & 1 & 0.4 & 1 & 1.7 \\
\hline Pericardial effusion & 1 & 0.4 & 1 & 1.7 \\
\hline Pleural effusion & 1 & 0.4 & 1 & 1.7 \\
\hline
\end{tabular}

Table 5 continued

\begin{tabular}{lccccc}
\hline & \multicolumn{2}{l}{ Any AEs } & & \multicolumn{1}{l}{$\begin{array}{l}\text { AEs requiring } \\
\text { hospitalization } \\
\text { in cycle 1 }\end{array}$} \\
\cline { 2 - 3 } \cline { 6 - 7 } & $n$ & $\%$ & & $n$ & $\%$ \\
\hline Pneumonia & 1 & 0.4 & & 1 & 1.7 \\
Pyrexia & 1 & 0.4 & & 0 & 0.0 \\
Uric acid increased & 1 & 0.4 & & 0 & 0.0 \\
Total & 226 & 100 & & 58 & 100 \\
\hline
\end{tabular}

$A E s$ adverse events, $C P K$ creatinine phosphokinase, $T$-Bil total bilirubin, $W B C$ white blood cell, $G G T$ gamma-glutamyltransferase, $C I P N$ chemotherapy-induced peripheral neuropathy, $A M Y$ amylase, $E C G$ electric cardiogram, $D V T$ deep vein thrombosis

The frequency of toxicity by study types was similar in both the dose-escalation and FIH studies $(12.4 \%$ and $10.4 \%$, respectively) (Table 4 ), possibly because FIH studies start at much lower dosage levels (e.g., $0.1 \mathrm{LD}_{10}$ of mice) compared to the doses used in dose-escalation studies. Severe toxicity in dose-finding studies was low (4.7\%), likely because the dose level in these studied may not reach the MTD. Consequently, in phase I oncology doseescalation and dose-finding studies, hospitalization during the first cycle should not be mandatory. In contrast, in FIH studies conducted to determine MTD, careful observation is required because the drug's toxicity profile is not well characterized, and hospitalization is one option to ensure patient safety. In FIH studies in Japan for drugs studied in earlier Western trials, it can be useful to use the Western clinical data to design phase I trials that reduce the risk of severe toxicity because it has been observed that the both the MTD and DLT frequency tends to be very similar between Western and Japanese patients [11].

The present study has some limitations. It is a retrospective study, and there is no analysis by design (e.g., conventional " $3+3$ " design, accelerated titration design, etc.) or by dosage levels. Of particular note, there is no standardization regarding the type of toxicity requiring hospitalization in Japan compared to other countries. We employed the definition described in the Methods, although it should be noted that hematological toxicity can be managed in an outpatient setting by blood transfusion with close monitoring in some cases. An effort between clinical investigators, regulatory authority, and other key stakeholders to identify a consensus definition of "toxicity requiring hospitalization" is warranted.

\section{Conclusion}

Our results indicate that there is a low rate of severe toxicity requiring hospitalization in phase I oncology trials in 
Japan. Consequently, the conventional approach requiring participants to be hospitalized during the first cycle of the phase I trial is not necessary. Rather, the outpatient setting should be considered for phase I clinical trials in Japan.

\section{Compliance with ethical standards}

Conflict of interest All authors have no conflict of interests to declare.

Open Access This article is distributed under the terms of the Creative Commons Attribution 4.0 International License (http://creativecommons.org/licenses/by/4.0/), which permits unrestricted use, distribution, and reproduction in any medium, provided you give appropriate credit to the original author(s) and the source, provide a link to the Creative Commons license, and indicate if changes were made.

\section{References}

1. ICH safety guidelines. http://www.ich.org/products/guidelines. $\mathrm{html}$

2. (2005) Guideline for clinical evaluation of anti-cancer drugs. In: Ministry of Health and Welfare
3. Hyman DM, Eaton AA, Gounder MM et al (2014) Nomogram to predict cycle-one serious drug-related toxicity in phase I oncology trials. J Clin Oncol 32(6):519-526

4. Postel-Vinay S, Gomez-Roca C, Molife LR et al (2011) Phase I trials of molecularly targeted agents: should we pay more attention to late toxicities? J Clin Oncol 29(13):1728-1735

5. Common terminology criteria for adverse events version 4.0. http://evs.nci.nih.gov/ftp1/CTCAE/About.html

6. Penta JS, Rosner GL, Trump DL (1992) Choice of starting dose and escalation for phase I studies of antitumor agents. Cancer Chemother Pharmacol 31(3):247-250

7. Le Tourneau C, Stathis A, Vidal L et al (2010) Choice of starting dose for molecularly targeted agents evaluated in first-in-human phase I cancer clinical trials. J Clin Oncol 28(8):1401-1407

8. Simon R, Freidlin B, Rubinstein L et al (1997) Accelerated titration designs for phase I clinical trials in oncology. J Natl Cancer Inst 89(15):1138-1147

9. Iasonos A, O'Quigley J (2014) Adaptive dose-finding studies: a review of model-guided phase I clinical trials. J Clin Oncol 32(23):2505-2511

10. Weber JS, Kahler KC, Hauschild A (2012) Management of immune-related adverse events and kinetics of response with ipilimumab. J Clin Oncol 30(21):2691-2697

11. Mizugaki H, Yamamoto N, Fujiwara Y et al (2015) Current status of single-agent phase I trials in Japan: toward globalization. J Clin Oncol 33(18):2051-2061 